\title{
Economic Impact of an Optimization-Based SCADA Model for an Office Building
}

\author{
Mahsa Khorram, Pedro Faria, Omid Abrishambaf, Zita Vale \\ GECAD - Knowledge Engineering and Decision Support Research Centre, Polytechnic of \\ Porto (IPP), Porto, Portugal \\ \{makgh, pnf, ombaf, zav\}@isep.ipp.pt
}

\begin{abstract}
The daily increment of electricity usage has led many efforts on the network operators to reduce the consumption in the demand side. The use of renewable energy resources in smart grid concepts became an irrefutable fact around the world. Therefore, real case studies should be developed to validate the business models performance before the massive production. This paper surveys the economic impact of an optimization-based Supervisory Control And Data Acquisition model for an office building by taking advantages of renewable resources for optimally managing the energy consumption. An optimization algorithm is developed for this model to minimize the electricity bill of the building considering day-ahead hourly market prices. In the case study, the proposed system is employed for demonstrating electricity cost reduction by using optimization capabilities based on user preferences and comfort level. The results proved by the performance of the system, which leads to having great economic benefits in the annual electricity cost.
\end{abstract}

Keywords: Energy Optimization, Renewable resources, SCADA.

\section{Introduction}

Nowadays, Demand Response (DR), and Renewable Energy Resources (RERs) are considered as major concepts in energy research topics [1]. The importance of DR programs and RERs is due to the advantages for all parts of the community including energy producers, consumers, and the environment by reducing the dependency on fossil fuels [2]. There is global concern about increasing of $\mathrm{CO} 2$ emissions, melting the glaciers, and the collapse of nature cycle [3], and hence, currently the demand of RERs and DR programs are increased [4]. In the DR programs, consumers are emboldened to change their electricity consumption pattern based on the variation of electricity price, or technical commands from the network operators [5]. DR programs can classify into two main incentive-based and price-based [6]. Real-Time Pricing (RTP), Time-Of-Use (TOU), and Critical-Peak Pricing (CPP) are included in the price-based programs. It

The present work was done and funded in the scope of the project UID/EEA/00760/2013 funded by FEDER Funds through COMPETE program and by National Funds through FCT. 
should be noted that in RTP, the used prices are the day-ahead or hour-ahead basis [6]. According to diverse surveys in [7], a large amount of energy consumption is dedicated to all types of buildings. In U.S. $35.5 \%$ of total electricity consumption belongs to the commercial buildings [8]. For taking advantages of DR programs, the buildings should be intelligent and equipped to several automation infrastructures [9]. Supervisory Control And Data Acquisition (SCADA) system is considered a part of DR implementation since it offers various advantages to have automatic load control in different types of buildings [10]. After equipping the buildings with the required infrastructures, an optimization algorithm is required to optimize and reduce the power consumption based on the existing conditions. For instance, in commercial buildings, Air Conditioners (ACs) and lighting systems can be considered as flexible and controllable loads [11], [12] due to their massive portion in electricity consumption [13].

This paper presents an optimization-based SCADA model, which is implemented in an office building. The main objective of the optimization algorithm is to minimize the electricity bill by using RERs and decreasing the power consumption according to dayahead hourly electricity prices. The power consumption of the ACs and lighting system have been selected to apply the consumption optimization. All the devices in this system are categorized based on the priorities defined by each user for each device in order to observe preferences and comfort level.

Several research works have been done in this context. In [14], the authors applied for DR programs in a residential building by using two types of retail pricing and control Heating, Ventilation, and Air Conditioning (HVAC) system. An optimal DR scheduling model for HVAC has been presented in [15], considering the thermal comfort of the users. [16] proposes an energy optimization controller algorithm which is used for industrial and commercial equipment such as HVAC, based on hour-ahead RTP programs. In [17], a SCADA system is implemented which is connected to MATLAB software to control and integrate different information of intelligent buildings such as temperature, ventilation, and illumination to manage and maintain the user satisfaction. In [18] represents a real implementation of an optimization model supported by a SCADA system, which employs several controlling and monitoring methods in order to manage the consumption and generation of the building. The main contribution of this paper is to survey the annual impact of the developed optimization algorithm in implemented SCADA system of an office building by considering dayahead electricity prices.

After this introductory section, the architecture of the developed model is described in Section 2. Section 3 represents a case study considered for the system, and the final results are presented in Section 4. Finally, section 5 details the main conclusions of the work.

\section{System Architecture}

This section describes the SCADA model which is implemented in an office building for managing energy consumption, and then, it focuses on an optimization algorithm employed in the SCADA for energy optimization purposes. 


\subsection{SCADA Model}

The SCADA model presented in this paper has been implemented in an office building, which includes eight offices, one server room, and a corridor. Every three offices have been categorized into a zone. Therefore, there are three independent zones including three offices. Only the relevant information regarding this SCADA system are mentioned in this section and more details are available on [18], which have been developed by the authors in the scope of their previous works.

There are three distributed based Programmable Logic Controllers (PLCs) for three zones, which control every three offices. Moreover, there is a main PLC associated with this model in order to supervise the other distributed based PLCs. In this paper, ACs and the lighting system of the building have been targeted for implementing the optimization purposes. The lighting system consists of 13 fluorescent lamps connected to the related PLC by Digital Addressable Lighting Interface (DALI). There are DALI ballasts installed for each lamp that allow the related PLC to fully control the intensity of the light as well as switching them ON/OFF. Furthermore, the SCADA system controls 9 ACs in the building. A microcontroller (Arduino ${ }^{\circledR}-$ www.arduino.cc), which equipped with an Ethernet Shield and an Infrared Light-Emitting Diode (IR LED) have been programmed and installed near to each AC. In fact, this controlling method emulates the remote control of ACs, somehow the SCADA takes decision for each $\mathrm{AC}$ and transmits the desired command to each $\mathrm{AC}$ controller (Arduino ${ }^{\circledR}$ ) via Ethernet interface (MODBUS protocol). Then, Arduino ${ }^{\circledR}$ controls the ACs based on SCADA decision.

In this SCADA model, there is a Photovoltaic (PV) system, which supplies a part of building consumption, and in high generation periods, the surplus of energy will inject into the utility grid. Also, six energy meters have been employed in the SCADA model to measure the consumption and generation of building. All energy meters follow serial communication with MODBUS-RTU (RS485) protocol to transmit the information to the related PLC.

Finally, a unit so-called Optimizer is connected to the model, which is responsible for solving optimization algorithm (explained in the next section) associated for the SCADA and provide the optimized information to the PLCs in order to be performed.

\subsection{Optimization Problem}

This section shows the details about the optimization algorithm, which is employed by developed SCADA system. This algorithm has been solved via "lpsolve" tools of RStudio optimization environment (www.rstudio.com) in order to minimize the electricity cost by managing the power consumption of the building. The objective of the optimization problem is to optimize the power consumption of the ACs a lighting system, based on the priority of each device, electricity cost variation, and stochastic PV generation. The power reduction of the ACs and lighting system depend profoundly on the electricity price, and the priority of each device. Fig. 1 shows the procedure of the optimization algorithm.

This optimization algorithm is a dynamic algorithm which runs online and updates the input parameters. As it can be seen in Fig. 1, the first part of the algorithm is dedicated to the definition of input data, such as total consumption of the building, total PV 
generation, consumption of the ACs, and consumption of the lighting system measured by SCADA system. In the meanwhile, the algorithm receives the information of the electricity market, since it is based on the day-ahead hourly electricity market. Then, the algorithm checks the amount of PV generation and compares that with the total consumption of ACs and lighting system. If the rate of consumption is greater than the PV generation, the algorithm continues to the next decision steps, otherwise, it will not enter into the optimization.

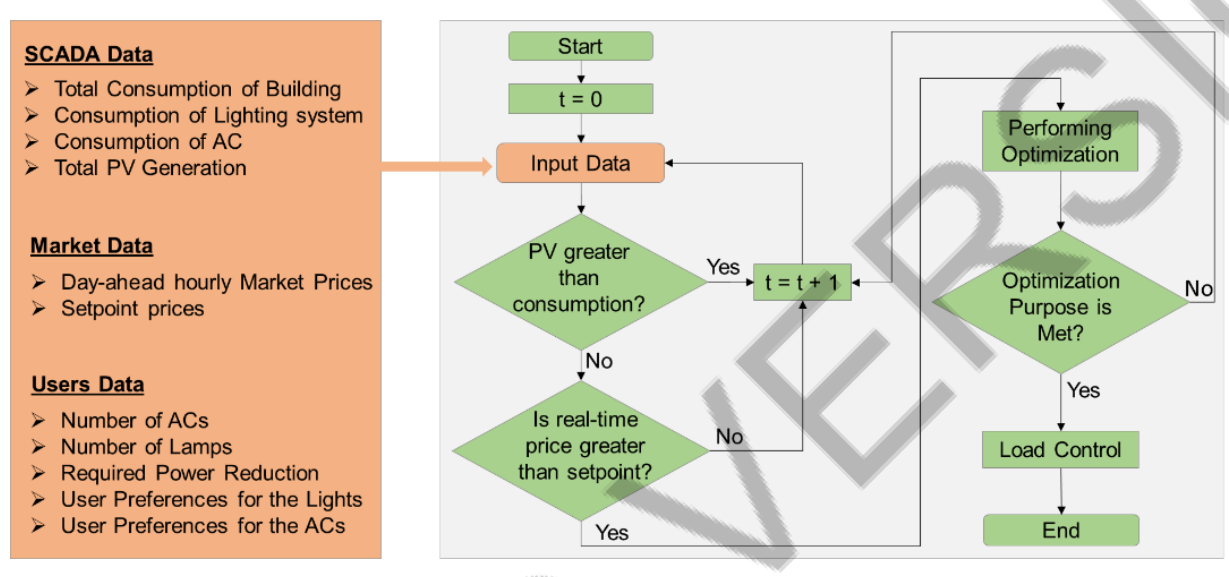

Fig. 1. Flowchart of the developed optimization algorithm.

The algorithm also receives the hourly electricity prices one day in advance and it takes an average value from all prices for the entire day and chooses it as a setpoint for the next day in order to perform the optimization. If the real-time electricity price is greater than the setpoint price, the algorithm starts optimizing the consumption, and in periods with a lower price than the setpoint, the optimization is not required, and the algorithm will check the input data again and again till it achieves the desired condition. By this way, consumption would be automatically optimized in the expensive periods.

Furthermore, there are some required data which should be indicated by the users, such as user preferences for the devices, the number of ACs, number of lights, and the amount of required reduction. User preferences can be interpreted as the priority of each device for the user. The weight of these priorities can be converted to a range between 0 and 1 . The priority numbers near 0 present the low priority devices from the user standpoint and the high priority devices have a number near to 1 . The objective function of the optimization problem is demonstrated in eq. (1):

Minimize

$$
\begin{gathered}
E C=\sum_{t=1}^{T}\left(\left(\sum_{a=1}^{A} P_{A C(a, t)} \times I_{A C(a, t)}+\sum_{l=1}^{L} P_{\operatorname{Lamp}(l, t)} \times I_{\operatorname{Lamp}(l, t)}\right)\right. \\
\left.-P V_{(t)}\right) \times \operatorname{COST}_{(t)}
\end{gathered}
$$


$P_{A C}$ and $P_{\text {Lamp }}$ indicate the real consumption of the AC devices and lighting system. $I_{A C}$ and $I_{L a m p}$ are the coefficients which present the importance of each AC, and each lamp respectively and $A$ and $L$ show the number of ACs and lamps. $P V$ stands for photovoltaic power generation and COST is the hourly electricity price in the time horizon of $t$.

There are several constraints that should be considered for the presented objective function. Equation (2) guarantees that the sum of power reduction of ACs $\left(P_{\text {Red.AC }}\right)$ and lighting system $\left(P_{\text {Red.Lamp }}\right)$ should be equal to the required power reduction $\left(P_{R R}\right)$. Equation (3) displays the technical limitations of power reduction from each $\mathrm{AC}$, which should be considered as a binary value since the ACs are classified in curtailment loads. Equation (4) limits the total power reduction of all AC devices, and (5) shows the technical limitation of each light individually. Equations (4), and (5) are considered to have a minimum illumination level for each lamp and also prevent turning all $\mathrm{AC}$ devices OFF in order to respect to the user comfort level. In fact, (5) models the lighting system as flexible loads, which allows the algorithm to reduce their consumption.

$$
\begin{gathered}
\sum_{a=1}^{A} P_{\text {Red.AC }(a, t)}+\sum_{l=1}^{L} P_{\text {Red.Lamp }(l, t)}=P_{R R(t)} \\
\forall t \in\{1, \ldots, T\} \\
P_{\text {Red.AC }(a, t)}=P_{\text {maxRed.AC }(a, t)} \times X \\
\forall X \in\{0,1\} \\
\forall t \in\{1, \ldots, T\} \\
\forall a \in\{1, \ldots, A\} \\
\sum_{a=1}^{A} P_{\text {Red.AC }(a, t)} \leq P_{\text {maxtotalRed.AC }(t)} \\
\forall t \in\{1, \ldots, T\} \\
0 \leq P_{\text {Red.Lamp }(l, t)} \leq P_{\text {maxRed.Lamp }(l, t)} \\
\forall t \in\{1, \ldots, T\} \\
\forall l \in\{1, \ldots, L\}
\end{gathered}
$$

$P_{\text {maxRed.AC }}$ declares the maximum power reduction for each AC device, and $P_{\text {maxTotalRed.AC }}$ is the maximum available power reduction from all AC devices. Furthermore, $P_{\text {maxRed.Lamp }}$ is the maximum power that is allowed to be reduced from each light. Required power reduction $\left(P_{R R}\right)$ is a flexible rate, which can be defined as any desired rate. However, in this paper, it is considered that the power reduction is the difference of the consumption of ACs and lighting system, and PV generation, in order to take advantages of PV generation in electricity bill. This definition is formulated by (6). In other words, (6) demonstrates that the PV generation supplies the consumption of ACs and lighting system as much as possible. Finally, (7) presents the limitation of importance coefficients. 


$$
\begin{gathered}
P_{R R(t)}=\left(\sum_{a=1}^{A} P_{A C(a, t)}+\sum_{l=1}^{L} P_{\operatorname{Lamp}(l, t)}\right)-P V_{(t)} \\
\forall t \in\{1, \ldots, T\} \\
0 \leq I_{A C(a, t)} \leq 1 ; 0 \leq I_{\operatorname{Lamp}(a, t)} \leq 1 \\
\forall t \in\{1, \ldots, T\} \\
\forall a \in\{1, \ldots, A\}
\end{gathered}
$$

As a summary, this section presented the technical specification of the SCADA model as well as the mathematical formulation of the proposed optimization algorithm. In the next section, this system is used for a case study in order to survey its annual economic impact.

\section{Case Study}

A case study is provided in this section to test and validate the functionalities of the developed system. For this purpose, the annual PV generation curve of the building and also annual consumption profiles of the ACs and lighting system are considered in order to perform the presented optimization problem. Fig. 2 illustrates the consumption and generation profiles considered for this case study. These curves are real data measured by the SCADA system and stored in a database.

The average consumption of each of $13 \mathrm{AC}$ is $1500 \mathrm{~W}$ and each of 9 lamps is $116 \mathrm{~W}$. The consumption curve showed in Fig. 2, is the consumption of ACs and lighting system for an entire year, and non-controllable loads are not shown in this figure. Furthermore, energy consumption in cold weather is higher than in hot. It means ACs are mostly used in winter for heating purposes.

Also, the generation curve showed in Fig. 2, is the real PV generation of the building for a year. This produced power will be first used for building consumption, and then in the high generation and low consumption periods, the energy surplus will be injected into the main grid. The electricity that is purchased from the main grid for the ACs and lighting system, is shown in Fig. 3.

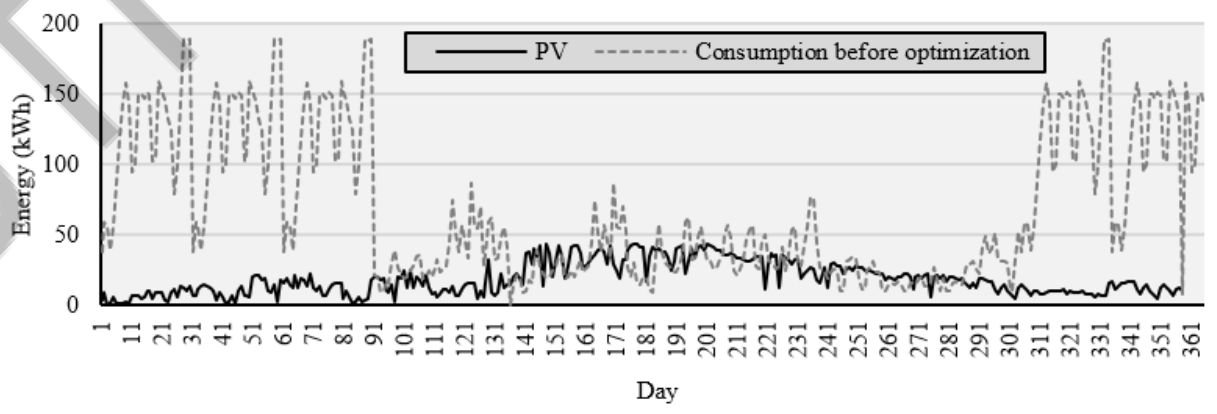

Fig. 2. Annual profiles considered for a case study for Consumption and PV generation. 
In the periods that optimization is required, the algorithm attempts to reduce the consumption profile shown in Fig. 3 (Power purchased from the main grid). In other words, the consumption curve illustrated in Fig. 3 is the data that should be provided to the algorithm as required consumption reduction. Although, a maximum reduction level always is considered by the algorithm in order to not switching all the devices OFF and keep high priority devices switched $\mathrm{ON}$ for respect to user preferences and comfort.

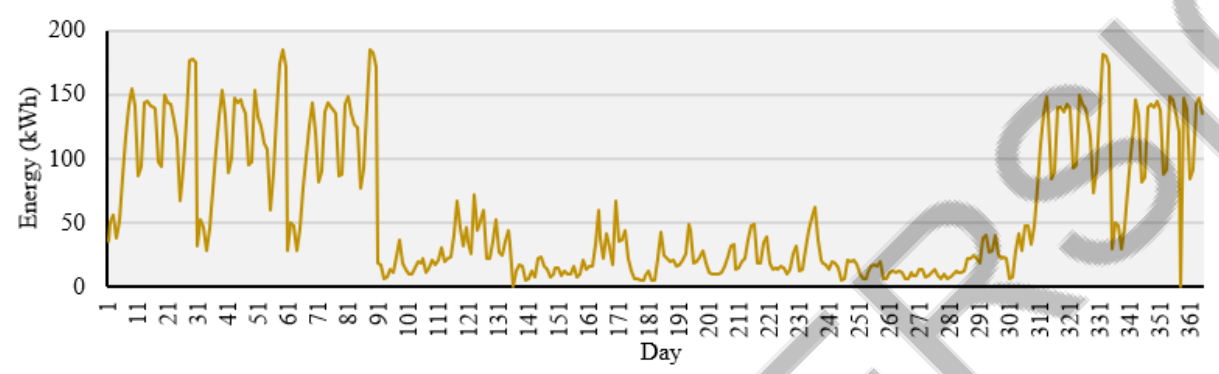

Fig. 3. Power purchased from the utility grid for ACs and lighting system electricity demand.

It should be noted that the prices used for defining setpoints, are for the entire year of 2016 and have been adapted from the Portuguese sector of Iberian Electricity Markets (MIBEL) [19].

\section{$4 \quad$ Results}

This section focuses on the results of the case study using the developed optimizationbased SCADA model for the office building. Fig. 4 illustrates the scheduled consumption profile after the optimization. In fact, the results that are shown in Fig. 4 is the output of the optimization algorithm regarding each AC and lamp.

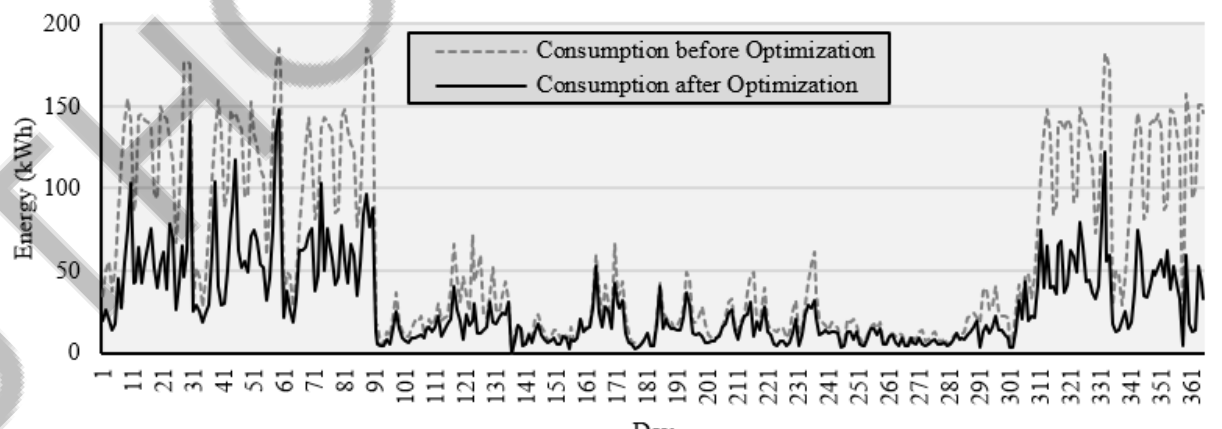

Day

Fig. 4. Consumption optimization results for ACs and lighting system in the office building.

The consumption profile that is shown by red lines in Fig. 4 is the purchased energy from the utility grid for ACs and lighting system consumption. Therefore, the 
optimization algorithm minimized this purchased energy based on the hourly prices in order to minimize the total energy cost (as it can be seen in Fig. 4 with blue line).

Furthermore, in the periods that the price is greater than the setpoint price, and the optimization algorithm is applied in the building, the user's comfort level is not violated since the algorithm minimizes the consumption of ACs and lighting system based on the priorities that each user defines. Also, the maximum consumption reduction level considered in the algorithm keeps the high priority devices always switched ON.

The other noticeable point in Fig. 4 is that from the beginning of the April to the end of October, the optimization algorithm is not widely performed since there is a significant amount of PV generation, and the generation supported all the demand for some periods and a reasonable part of it in other periods.

In order to survey the economic impact, Fig. 5 illustrates the accumulated cost for an entire year under three different conditions.

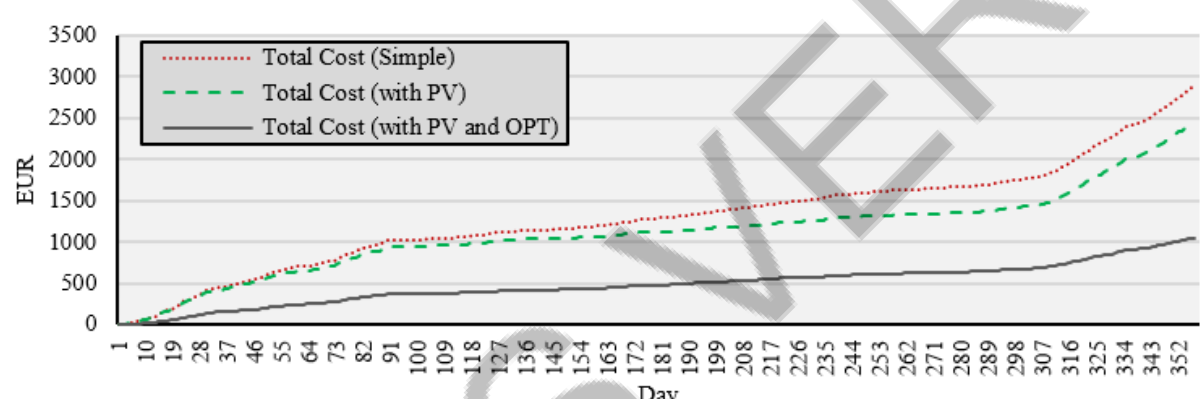

Fig. 5. Accumulated energy costs for one year considering three scenarios.

As it can be seen in Fig. 5, the red line indicates the purchased energy cost from the main grid for the ACs and lighting system while no PV and optimization capabilities are considered for the SCADA. In fact, this scenario is considered as a basis to be compared with the other scenarios.

The gap between the red and green lines in Fig. 5 indicates the cost that is gained due to the local PV generation. This gap is increased during the summer, where the PV generation is higher, and it supplies a great part of building consumption. Finally, by employing both PV system and optimization capabilities, the total electricity cost of the building is reduced significantly, which indicates the advantages of an optimizationbased SCADA model equipped with a local energy resource (e.g. PV).

In order to clarify the annual cost comparison, Table 1 demonstrates the details of annual electricity costs for the presented scenarios.

Table 1. Annual cost comparison for three scenarios.

\begin{tabular}{lll}
\hline \multicolumn{1}{c}{ Scenario } & Annual cost (EUR) & Cost reduction (Compared to simple) \\
\hline Simple (No PV, No OPT) & 2890.92 & - \\
With PV, No OPT & 2455.49 & $15.06 \%$ \\
With PV and OPT & 1048.99 & $63.71 \%$ \\
\hline
\end{tabular}


As it is clear in Table 1, the amount of cost reduction with a PV system and without optimization capability is $15.06 \%$ compared to the simple scenario. While both the PV system and optimization are used by the SCADA, the cost is $63.71 \%$ is reduced compared to the simple scenario. This proves the functionalities and capabilities of the developed optimization-based SCADA system, which presents an acceptable performance in day-ahead and real-time electricity markets.

\section{Conclusions}

Electricity demand increment in the current state of energy system requires more survey and investigation regarding energy optimization and using renewable energy resources. Commercial buildings, especially office buildings are appropriate targets for optimizing the lighting systems and air conditioner devices. However, the need for real case studies is obvious for testing the business model before massive production.

This paper surveyed the economic impacts of a real optimization-based SCADA model implemented for an office building to make decisions and manage the consumption based on the day-ahead electricity market. Moreover, an optimization algorithm was developed for the SCADA system in order to minimize the electricity bill based on the priorities defined by each office user for each air conditioner and lighting system.

The results of the case study validated the functionalities of the presented optimization-based SCADA model in the day-ahead electricity market. Furthermore, a comparison of annual electricity costs of the building was demonstrated by considering different scenarios to show the economic benefits of applying the proposed optimization algorithm.

\section{References}

1. Arun, S., Selvan, M.: Intelligent Residential Energy Management System for Dynamic Demand Response in Smart Buildings. IEEE Systems Journal 12(2), 1329-1340 (2018).

2. Khorram, M., Faria, P., Vale, Z.: Optimization-Based Home Energy Management System Under Different Electricity Pricing Schemes. In: IEEE 16th International Conference of Industrial Informatics (IEEE INDIN), (2018).

3. Rotger-Griful, S., Welling, U., Jacobsen, R: Implementation of a building energy management system for residential demand response. Microprocessors and Microsystems 55, 100110 (2017).

4. Rehmani, M., Reisslein, M., Rachedi, A., Erol-Kantarci, M., Radenkovic, M.: Integrating Renewable Energy Resources Into the Smart Grid: Recent Developments in Information and Communication Technologies. IEEE Transactions on Industrial Informatics 14(7), 28142825 (2018).

5. Abrishambaf, O., Faria, P., Gomes, L., Spínola, J., Vale, Z., Corchado, J.: Implementation of a Real-Time Microgrid Simulation Platform Based on Centralized and Distributed Management. Energies 10(6), 806-820, (2017).

6. Faria, P., Vale, Z.: Demand response in electrical energy supply: An optimal real time pricing approach. Energy 36(8), 5374-5384 (2011). 
7. Yang, F., Guo, Q., Pan, Z., Sun, H.: Building energy management based on demand response strategy considering dynamic thermal characteristic. In: IEEE Power \& Energy Society General Meeting, (2017).

8. Yu, L., Xie, D., Jiang, T., Zou, Y., Wang, K.: Distributed Real-Time HVAC Control for Cost-Efficient Commercial Buildings Under Smart Grid Environment. IEEE Internet Things Journal 5(1), 44-55 (2018).

9. Tsui, K., Chan, S.: Demand Response Optimization for Smart Home Scheduling Under Real-Time Pricing. IEEE Transaction on Smart Grid 3(4), 1812-1821 (2012).

10. Khorram, M., Faria, P., Abrishambaf, O., Vale, Z.: Demand Response Implementation in an Optimization Based SCADA Model Under Real-Time Pricing Schemes. In: 15th International Conference on Distributed Computing and Artificial Intelligence (DCAI), (2018).

11. Faria, P., Pinto, A., Vale, Z., Khorram, M., de Lima Neto, F., Pinto, T.: Lighting consump tion optimization using fish school search algorithm. In: 2017 IEEE Symposium Series on Computational Intelligence (SSCI), (2017).

12. Pan, X., Lee, B.: An Approach of Reinforcement Learning Based Lighting Control for Demand Response. In PCIM Europe 2016, Nuremberg, Germany, (2016).

13. Shi, J., Yu, N., Yao, W.: Energy Efficient Building HVAC Control Algorithm with Realtime Occupancy Prediction. Energy Procedia 111, 267-276, (2017).

14. Yoon, J., Bladick, R., Novoselac, A.: Demand response for residential buildings based on dynamic price of electricity. Energy and Building, 80, 531-541, (2016).

15. Kim, Y.: Optimal Price-Based Demand Response of HVAC Systems in Multi-Zone Office Buildings Considering Thermal Preferences of Individual Occupants. IEEE Transactions on Industrial Informatics, 1-1 (2018).

16. Abdulaal, A., Asfour, S.: A linear optimization based controller method for real-time load shifting in industrial and commercial buildings. Energy and Building, 110, 269-283 (2016).

17. Figueiredo, J., Costa, J.: A SCADA system for energy management in intelligent buildings. Energy and Building, 49, 85-98 (2012).

18. Khorram, M., Abrishambaf, O., Faria, P., Vale, Z.: Office building participation in demand response programs supported by intelligent lighting management. Energy Informatics, 1(9), 1-14 (2018).

19. MIBEL Electricity Market, http://www.omip.pt, last accessed 2018/07/08. 